Anales de Geografía de la Universidad Complutense ISSN: 0211-9803

http://dx.doi.org/10.5209/AGUC.66947

\title{
Análisis de la distribución territorial de los alojamientos rurales y convencionales en los núcleos rurales de Extremadura ${ }^{1}$
}

\author{
Juan Ignacio Rengifo Gallego²; José Manuel Sánchez Martín ${ }^{3}$ \\ Recibido: 28 de diciembre del 2017 / Enviado a evaluar: 14 de enero del 2018 / Aceptado: 17 de octubre del 2019
}

Resumen. Extremadura cuenta con 388 municipios, distribuidos entre las provincias de Badajoz (165) y Cáceres (223). Por la mayor parte de estos municipios se ha ido implantando una red de alojamientos rurales a partir del año 1992, fecha en la que se publicó el primer Decreto que regulaba esta tipología de alojamientos. En la actualidad, la normativa establece que los alojamientos rurales pueden ubicarse en aquellos municipios que no sobrepasen los 10.000 habitantes. Este límite posibilita que dichos alojamientos se puedan implantar en el 96,6\% de los municipios de Extremadura. Teniendo en cuenta estas premisas, este artículo tiene como objetivo medir y analizar la distribución de los alojamientos rurales, en comparación con los convencionales, en estos municipios. Los resultados muestran una realidad en la que hay divergencias entre los dos bloques de municipios establecidos en el trabajo: los de menos de 2.000 habitantes y los que tienen entre 2.000 y 10.000 habitantes.

Palabras clave: Extremadura; turismo rural; alojamientos rurales; distribución territorial; núcleos rurales.

\section{[en] Analysis of the distribution of rural and conventional tourism accommodation providers in the rural towns of Extremadura}

\begin{abstract}
Extremadura comprises 388 towns and villages - 165 in the province of Badajoz and 223 in the province of Cáceres. Most of these have steadily set up a network of rural tourism businesses starting in 1992, when the first piece of legislation regulating this type of accommodation providers (Decreto) was passed. At present, regulations dictate that rural accommodation businesses can be opened in towns smaller than 10,000 inhabitants. This population limit actually implies that these businesses can be set up
\end{abstract}

1 La presente publicación forma parte de los estudios correspondientes al proyecto de investigación "Diseño y elaboración de productos estratégicos diferenciados para la potenciación del turismo rural en Extremadura. De la detección de problemas a la propuesta de soluciones basadas en criterios geoestadísticos", cuya clave es IB 16040, y se encuentra financiado por la Consejería de Economía e Infraestructuras de la Junta de Extremadura y por el Fondo Europeo de Desarrollo Regional (FEDER).

2 Universidad de Extremadura.

E-mail: irengifo@unex.es

3 Universidad de Extremadura.

E-mail: jmsanche@unex.es 
in $96.6 \%$ of municipalities in Extremadura. Based on these principles, this paper seeks to measure and analyse the distribution of rural accommodation businesses, by contrast to conventional accommodation providers, in these towns. Results indicate that there are striking differences between the two groups of small towns established for our research: those with fewer than 2,000 inhabitants and those ranging between 2,000 and 10,000 inhabitants.

Keywords: Extremadura; rural tourism; rural accommodation; rural areas; geographical distribution.

\section{[fr] Analyse de la distribution du territoire des logements ruraux et conventionnels dans les zones rurales de l'Estrémadure}

Résumé. L'Estrémadure compte 388 municipalités distribuées entre les provinces de Badajoz (165) et de Cáceres (223). Dans la plupart de ces communes, un réseau de logements ruraux a été mis en place depuis 1992, date à laquelle le premier décret réglementant ce type d'hébergement a été publié. Actuellement, les règlements établissent que les logements ruraux peuvent être situés dans les municipalités qui ne dépassent pas les 10.000 habitants. Cette limite permet que ces logements ruraux puissent être mis en place dans 96,6\% des municipalités d'Estrémadure. Considérant les données citées, cet article vise à mesurer et analyser la répartition des logements ruraux, en comparaison avec les logements conventionnels dans ces municipalités. Les résultats montrent une réalité dans laquelle il existe des divergences entre les deux blocs de municipalités établis dans le travail: celles qui ont moins de 2.000 habitants et celles qui ont entre 2.000 et 10.000 habitants.

Mots clés: Logements ruraux; hébergements conventionnels; centres ruraux; distribution du territoire; analyse.

Cómo citar. Rengifo Gallego, J.I. y Sánchez Martín, J.M. (2019): Análisis de la distribución territorial de los alojamientos rurales y convencionales en los núcleos rurales de Extremadura. Anales de Geografía de la Universidad Complutense, 39(2), 463-484.

Sumario. 1. Introducción. 2. Objetivos y metodología. 3. Los alojamientos turísticos en Extremadura. 3.1. Marco Normativo general. 3.2. Alojamientos convencionales. 3.3. Alojamientos rurales. 4. Resultados. 4.1. Número y capacidad de los alojamientos convencionales hoteleros. 4.2. Número y capacidad de los alojamientos convencionales extrahoteleros. 4.3. Número de alojamientos rurales. 5. Conclusiones. 6. Bibliografía.

\section{Introducción}

Extremadura es la quinta Comunidad Autónoma por superficie de España, con algo más de $41.600 \mathrm{~km}^{2}(8,22 \%$ de la superficie total española) distribuidos por las dos provincias más extensas del país. Su territorio está dividido administrativamente en 388 municipios (año 2016) que vienen a representar el 4,77\% de los existentes a escala nacional. Esta cifra solo se ve superada por el número de municipios con que cuentan las Comunidades Autónomas de Andalucía, Aragón, Castilla y León, Castilla la Mancha, Cataluña y Comunidad Valenciana.

La distribución de los municipios extremeños, tal y como muestra la Tabla 1, refleja una realidad carente de equilibrio en dos ámbitos: territorial y demográfico. En consecuencia, la provincia de Cáceres cuenta con un mayor número de municipios, $57,4 \%$, mientras que la provincia de Badajoz alberga al 42,6\% restante (casi 15 puntos de diferencia). Por su parte, el tipo de municipio predominante, de acuerdo 
con su tamaño, se corresponde con el rango de menos de 2.000 habitantes que, en el caso de la provincia de Cáceres, llega a sumar al $87 \%$ del total de municipios. En sentido contrario, los municipios mayores de 10.000 habitantes representan, tan solo, el 3,4\% del total, siendo Badajoz la provincia que acumula la mayor parte de ellos (nueve de trece). Sin embargo, el reparto de la población en función del tamaño de los municipios responde a criterios diferentes debido a que son los mayores de 10.000 habitantes los que, a pesar de su escaso número, concentran el $40 \%$ de la población absoluta extremeña.

Tabla 1. Distribución de municipios en función del tamaño. Año 2016.

\begin{tabular}{|c|c|c|c|c|c|c|c|c|}
\hline \multirow{2}{*}{ Provincias } & \multicolumn{2}{|c|}{$\begin{array}{l}\text { Municipios } \\
<2.000 \text { habs. }\end{array}$} & \multicolumn{2}{|c|}{$\begin{array}{c}\text { Municipios } \\
2.001 \text { a } 9.999 \text { habs. }\end{array}$} & \multicolumn{2}{|c|}{$\begin{array}{c}\text { Municipios } \\
>10.000 \text { habs. }\end{array}$} & \multicolumn{2}{|c|}{ Total } \\
\hline & $\mathrm{N}^{\mathrm{o}}$ & $\%$ & $\mathrm{~N}^{\mathrm{o}}$ & $\%$ & $\mathrm{~N}^{\mathrm{o}}$ & $\%$ & $\mathrm{~N}^{\mathrm{o}}$ & $\%$ \\
\hline Badajoz & 99 & 60 & 57 & 34,5 & 9 & 5,5 & 165 & 100 \\
\hline Cáceres & 194 & 87 & 25 & 11,2 & 4 & 1,8 & 223 & 100 \\
\hline Total & 293 & 75,5 & 82 & 21,1 & 13 & 3,4 & 388 & 100 \\
\hline
\end{tabular}

Fuente: INE. Elaboración propia.

Esta caracterización, que otorga un perfil acentuadamente rural al territorio regional, se ve reforzada por la baja densidad demográfica de la inmensa mayoría de los municipios extremeños. En este sentido, hay que tener en cuenta que la delimitación del medio rural se basa, en gran parte, en la consideración de estos factores, tal y como expresa Sancho y Reinoso (2012) y manifiestan en sus marcos definitorios del medio rural la European Commission (1997), la OCDE (1994) o la Ley estatal 45/2007 de 13 diciembre, para el Desarrollo Sostenible del Medio Rural. Esta Ley, en su artículo 3, define al medio rural como "el espacio geográfico formado por la agregación de municipios o entidades locales menores definido por las administraciones competentes que posean una población inferior a 30.000 habitantes y una densidad inferior a los 100 habitantes por $\mathrm{km}^{20}$.

Profundizando en la cuestión de la ya apuntada densidad poblacional de la región extremeña, hay que señalar que esta es muy baja, al situarse, por término medio, en los $26,11 \mathrm{hab} / \mathrm{km}^{2}$. Este dato indica que Extremadura está muy alejada de la densidad media nacional $\left(92 \mathrm{hab} / \mathrm{km}^{2}\right)$ y de la densidad media que alcanzan los países que conforman la Unión Europea $\left(116 \mathrm{hab} / \mathrm{km}^{2}\right)$. Además, son numerosos los municipios extremeños que están por debajo de la media regional, tal y como puede verificarse en la Figura 1.

De la lectura visual de este mapa se desprende la existencia de un inmenso territorio, conformado por 279 municipios extremeños, en los que la densidad de habitantes es inferior a la media regional $\left(30.637,72 \mathrm{~km}^{2}\right.$, es decir, el $73,5 \%$ de la superficie total de la región), y, en cuyo ámbito territorial viven 313.397 personas. Por otra parte, también se aprecia la minoritaria superficie englobada por los 109 municipios en los que se supera esta media $\left(11.027 \mathrm{~km}^{2}\right.$, es decir, el $26,5 \%$ del territorio regional) y en la que se asientan 774.381 habitantes. 
Bajo estas condiciones, es muy fácil entender que se venga identificando a Extremadura como un laboratorio perfecto para la puesta en marcha de iniciativas que propicien el desarrollo de los municipios rurales, a sabiendas de que en estos municipios existen graves problemas sociales y económicos que pasan por la consabida pérdida de población, escasa diversificación económica, envejecimiento, masculinización y menor renta disponible de sus habitantes, salvo contadas excepciones. Por tanto, si se tienen en cuenta estas premisas, parece lógico que tradicionalmente se haya considerado al turismo como una actividad con facultades para dinamizar el medio rural, hipótesis que ha sido avalada, con diferentes resultados, por algunos análisis realizados en los últimos años. No obstante, no se puede ignorar que en no pocas ocasiones se ha magnificado el papel que podía jugar el turismo como elemento dinamizador del medio rural, hasta el punto de que se le atribuyera el carácter de remedio contra la mayor parte de los males que padece el mundo rural, si bien con el paso de los años se ha visto que esto no ha sido así.

Figura 1. Densidad de habitantes en Extremadura a escala municipal. Año 2016.

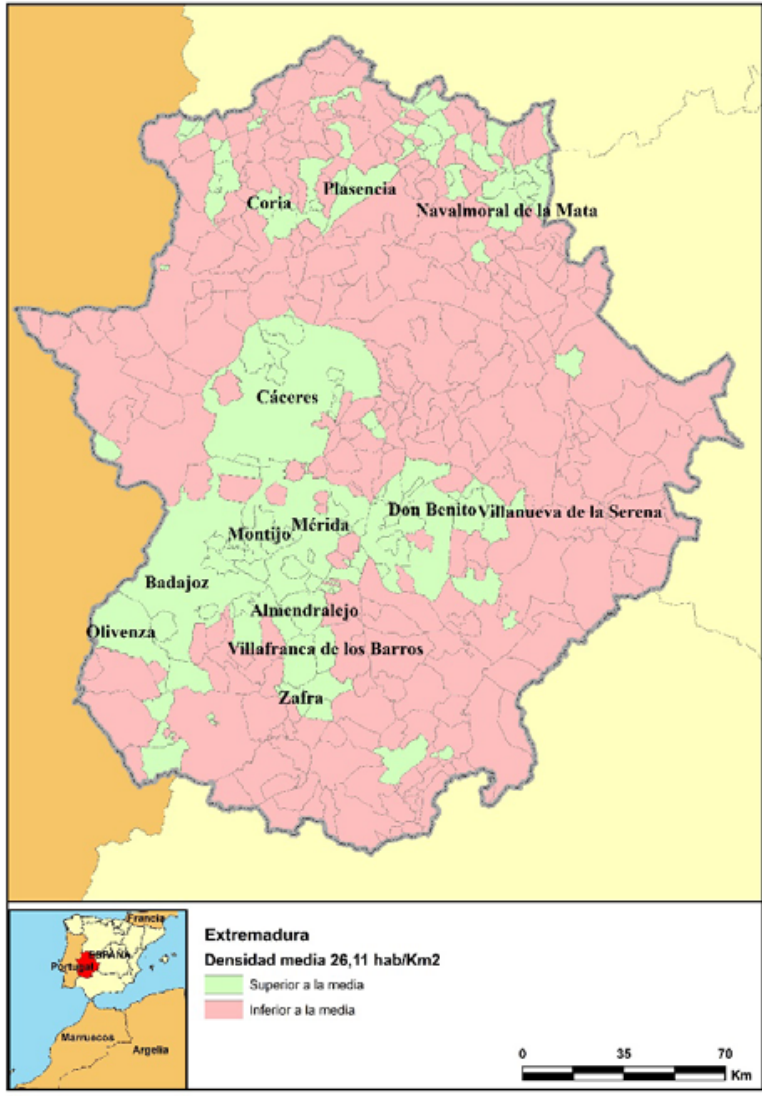

Fuente: Elaboración propia. 
Con estas premisas, cuando se reguló una planta de alojamientos rurales con carácter específico en Extremadura, a principios de la década de los noventa, todo hacía presagiar que se estaba apostando por algo exitoso, máxime cuando el medio rural acumulaba una ingente cantidad de recursos patrimoniales (histórico-culturales y naturales) en los que se venía invirtiendo dinero público con la finalidad de promocionarlos, conservarlos y hacer posible el uso y disfrute social.

Con el paso del tiempo se ha constatado un irrefutable éxito en lo que a crecimiento del número de alojamientos rurales se refiere (Rengifo et al., 2013, Campesino et al., 1997 y 2016), si bien no se puede ignorar que este incremento se ha visto acompañado no solo de luces, sino también de sombras (Rengifo y Sánchez, 2016). Como resultado de este crecimiento se han obtenido evidentes repercusiones sociales, económicas y territoriales en Extremadura, a las que la comunidad científica le ha prestado especial atención a través de la realización de diferentes trabajos en los se han abordado temáticas muy diversas: análisis descriptivo y crítico (Alvarado, 2006; Sánchez y Rengifo, 2016) modelos de crecimiento y distribución territorial (Rengifo et al.2013; Rengifo y Sánchez, 2016), evaluación del potencial turístico y adecuación de la planta de alojamientos rurales al potencial turístico (Sánchez et al., 2013, Sánchez et al. 2014; Sánchez et al., 2017) y tratamiento mediante técnicas SIG (Sánchez et al., 2001 y Sánchez, 2009).

Sin embargo, y a pesar de la literatura generada sobre el espectacular desarrollo que ha experimentado la planta turística de los alojamientos rurales de Extremadura, se ha investigado de forma muy escasa sobre si este crecimiento ha corrido paralelo al que se ha producido en la planta turística convencional en los mismos municipios. En este sentido, a los efectos de este trabajo se entiende que son alojamientos convencionales todos aquellos que no están incluidos en la normativa específica de alojamientos rurales, sean de carácter hotelero (hoteles, hostales, pensiones) o extrahotelero (campamentos de turismo, albergues y apartamentos turísticos). La principal diferencia entre los alojamientos rurales y convencionales se relaciona con dos hechos fundamentales: En primer lugar, a los llamados alojamientos convencionales no se les exige ningún requisito relacionado con el número de habitantes que debe reunir el núcleo de población en el que desean implantarse, contrariamente a lo especificado en los alojamientos rurales $\mathrm{y}$, en segundo lugar, no se ven limitados de forma alguna por la normativa a contar con un número máximo de habitaciones ni a responder a unas características arquitectónicas determinadas.

\section{Objetivos y metodología}

El objetivo principal de este trabajo se centra en analizar y comparar, para después interpretar, cuáles son las tipologías de alojamientos (convencionales o rurales) más representativas en los municipios rurales de Extremadura, teniendo en cuenta el límite de 10.000 habitantes que en su momento estableció el Decreto 65/2015 sobre la ordenación y sistema de clasificación de los alojamientos de turismo rural. No obstante, hay que decir que una reciente modificación de la Ley de Turismo del año 
2011 (Ley 6/2018), permite la ubicación de alojamientos rurales en municipios de hasta 20.000 habitantes que, a los efectos de este artículo, no se ha tenido en cuenta ya que los datos utilizados son del año 2017, fecha en la que esta última medida no estaba en vigor.

El periodo temporal elegido para confirmar la hipótesis es el comprendido entre los años 2001 y 2017, años en los que tiene lugar el crecimiento más intenso de los alojamientos rurales en Extremadura.

El proceso metodológico seguido en el trabajo se ve reflejado en su estructura, tal y como a continuación se expone:

- En el epígrafe introductorio se acomete la tarea de explicar la distribución del poblamiento en Extremadura a escala municipal, estableciendo tres agrupaciones que concuerdan con los objetivos que se persiguen en este trabajo. Por este motivo se procede a separar los municipios en función de su tamaño (peso demográfico): municipios con una población inferior a 2.000 habitantes, municipios con un peso demográfico entre 2.000 y 9.999 habitantes y municipios que superan la barrera de los 10.000 .

- En el punto 2 se hace un repaso de la normativa vigente para establecer la pertinente separación entre alojamientos convencionales y rurales. Para ello se ha analizado la normativa comprendida entre el año 1992 y el año 2018.

- En el punto tercero se muestran los resultados obtenidos, tomando como fuente los datos oficiales recabados de la Dirección General de Turismo de la Junta de Extremadura. Se utilizan estos datos por su fiabilidad, al ser dicha Dirección General el organismo competente a la hora de autorizar la apertura de un establecimiento. Los resultados se muestran en tablas, agrupados de acuerdo con los rangos antes establecidos en función del peso demográfico de los municipios y, con posterioridad, se han cartografiado a escala municipal. Para la cartografía se ha recurrido a la base proporcionada por el Instituto Geográfico Nacional (IGN), correspondiente a la Base Topográfica Nacional a escala 1:100.000 en su versión de 2017.

- En el último punto se extraen las conclusiones oportunas.

Por último, hay que añadir que el presente trabajo se justifica por la inexistencia de un análisis que vaya en el sentido planteado, al centrarse los trabajos publicados, hasta el momento, en el estudio de los alojamientos rurales de forma individualizada, sin tener en cuenta el peso que tienen el resto de alojamientos que se distribuyen por los municipios seleccionados.

\section{Los alojamientos turísticos en Extremadura}

\subsection{Marco Normativo general}

La Ley de Turismo de Extremadura en vigor fue aprobada en el año 2011 (Ley 2/2011, de 31 de enero, de desarrollo y modernización del turismo de Extremadura), aunque con posterioridad fue levemente modificada en el año 2014 por la Ley 7/2014, de 5 de agosto, de modificación de la Ley 2/2011, de 31 de enero, de desarrollo y 
modernización del turismo en Extremadura y en fechas más cercanas por la Ley 6/2018, de 12 de julio, de modificación de la Ley 2/2011, de 31 de enero, de desarrollo y modernización del turismo de Extremadura. Estas leyes derogaron a la anterior que procedía del año 1997. En la Ley vigente, dentro del Título II, en su Capítulo I, artículo 39, se establecen cuatro tipologías de empresas turísticas:

- De alojamiento turístico. Son empresas de alojamiento turístico aquellas que se dedican, de manera profesional y habitual, mediante contraprestación económica, a proporcionar al turista habitación o residencia, con o sin prestación de otros servicios.

- De restauración. Son aquellas que se dedican de forma habitual y profesional, a suministrar desde establecimientos abiertos al público, mediante contraprestación económica, comidas y/o bebidas para consumir en el propio establecimiento o fuera de él.

- De intermediación turística. Son empresas de intermediación turística aquellas que se dedican profesional y habitualmente al ejercicio de actividades de información, mediación y organización de servicios turísticos, utilizando medios propios o ajenos para llevarlas a cabo.

- De actividades turísticas alternativas. Las empresas de actividades turísticas alternativas son aquellas que ofertan la práctica de actividades turísticas tales como deportivas, medioambientales, de agroturismo, turismo activo, turismo ornitológico, culturales, formativas, recreativas o de ocio, belleza, salud, y aquellas otras que con su actividad contribuyan a la diversificación y mejora de la oferta turística.

Tabla 2. Empresas de alojamiento turístico

\begin{tabular}{|l|l|l|}
\hline \multicolumn{1}{|c|}{ Alojamientos Hoteleros } & Alojamientos Extrahoteleros & Alojamientos Rurales \\
\hline Hoteles & Apartamentos Turísticos & Hoteles Rurales \\
Hoteles-Apartamentos & Albergues turísticos & Casas Rurales \\
Hostales & Campamentos de turismo & \\
Pensiones & Zonas de acampada & \\
& Cualquier otro que se fije & \\
& reglamentariamente & \\
\hline
\end{tabular}

Fuente: Ley de Turismo 2/2011. Diario Oficial de Extremadura (DOE)

De estas cuatro tipologías son de interés para este trabajo las empresas de alojamiento turístico que, de acuerdo con lo reflejado en los artículos 57 a 69 de la Ley, se clasifican, tal y como se aprecia en la Tabla 2, en tres modalidades diferentes: Alojamiento hotelero, alojamiento extrahotelero y alojamiento rural. Dentro de cada una de estas tres modalidades, a su vez, se establecen diferentes clases y categorías que son enumeradas parcialmente en la siguiente tabla y tratadas de forma específica en los epígrafes siguientes. 


\subsection{Alojamientos convencionales}

Como ya se ha advertido, en este trabajo se consideran alojamientos convencionales los que aparecen incluidos en los bloques de los alojamientos extrahoteleros y hoteleros.

Los alojamientos hoteleros están ordenados y clasificados por lo expresado en el Decreto 235/2012, de 4 de diciembre, por el que se establece la ordenación y clasificación de los Alojamientos Turísticos Hoteleros de la Comunidad Autónoma de Extremadura. Este Decreto, de conformidad con lo reflejado en la Ley antes mencionada, establece la siguiente clasificación:

- Hoteles y hoteles-apartamentos, que cuentan con cinco categorías identificadas por estrellas. En el Decreto se establecen, asimismo, especializaciones para algunos casos, como por ejemplo para los hoteles balnearios.

- Hostales, agrupados en dos categorías identificadas por estrellas.

- Pensiones, encuadradas en una única categoría.

Por su parte, los alojamientos extrahoteleros aparecen ordenados y clasificados reglamentariamente por diferentes decretos, según los casos:

- Campamentos de turismo: Decreto 170/1999, de 19 de octubre, por el que se regulan los Campamentos Públicos de Turismo, Campamentos Privados y Zonas de Acampada Municipal.

- Apartamentos turísticos: Decreto 182/2012, de 7 de septiembre, por el que se establece la ordenación y clasificación de los apartamentos turísticos de la Comunidad Autónoma de Extremadura

- Albergues turísticos: Decreto 244/2012, de 18 de diciembre, por el que se establece la ordenación de los Albergues Turísticos de la Comunidad Autónoma de Extremadura.

En síntesis, el conjunto de alojamientos hoteleros y extrahoteleros existentes en Extremadura conformaría lo que a efectos de este trabajo se considera alojamientos convencionales, cuya trayectoria histórica y evolución es muy desigual dependiendo de las diferentes figuras.

\subsection{Alojamientos rurales}

Por su singularidad e interés para este trabajo, se procede inicialmente a describir el proceso diacrónico comprendido entre el momento en el que surge la primera regulación normativa de estos alojamientos en Extremadura, año 1992, hasta la actualidad. Sobre los alojamientos rurales hay que decir que han sido la base del crecimiento del turismo rural en Europa, al margen de las fórmulas o denominaciones utilizadas en cada caso (Cánoves et al., 2005).

Los alojamientos rurales empiezan a desarrollarse en la región tras la publicación de un primer decreto (Decreto 132/1992, de 15 de diciembre, por el que se crea y regula la modalidad de alojamiento en el medio rural y servicios turísticos complementarios en la Comunidad Autónoma de Extremadura), que marcó el punto 
de partida del desarrollo de estos alojamientos específicos, si bien no hay que ignorar la existencia de experiencias previas a escala nacional. En este sentido, hay que mencionar el programa de vacaciones en Casas de Labranza puesto en marcha en España en la década de los 60 que, de acuerdo con lo expresado por Carazo (1982), iba "dirigido a fomentar y facilitar la participación de los agricultores en la utilización económica de sus recursos turísticos, proporcionando simultáneamente a amplios sectores de población urbana una forma de turismo de calidad a costes muy razonables".

Con estos antecedentes, y tras la publicación del Decreto del año 1992 en Extremadura, lo cierto es que los alojamientos rurales han ido experimentando cambios sustanciales a lo largo de los últimos 25 años. Estos cambios aparecen reflejados, con claridad, en los sucesivos decretos que se han ido aprobando en los últimos años:

- Decreto 132/1992, de 24 de diciembre, por el que se crea y regula la modalidad de alojamiento en el medio rural y servicios turísticos complementarios en la Comunidad Autónoma de Extremadura. Publicado en el DOE número 100 de 24 de diciembre de 1992.

- Decreto 125/1998, de 6 de octubre, de ordenación del alojamiento turístico en el medio rural. Publicado en el DOE número 117 de 13 de octubre de 1998.

- Decreto 87/2007, de 8 de mayo, de ordenación y clasificación del alojamiento turístico en el medio rural. Publicado en el DOE número 55 de 15 de mayo de 2007.

- Decreto 204/2012, de 15 de octubre, por el que se establece la ordenación y clasificación de los alojamientos de turismo rural de la Comunidad Autónoma de Extremadura. Publicado en el DOE número 202 de 18 de octubre de 2012.

- Decreto 65/2015 de 14 de abril, por el que se establece la ordenación y sistema de clasificación de los alojamientos de turismo rural de la Comunidad Autónoma de Extremadura. Publicado en el DOE número 74 de 20 de abril de 2015.

Los cambios introducidos por los sucesivos decretos y Leyes, como ya ha sido analizado de forma específica por Rengifo y Sánchez (2016), han sido sustanciales y se pueden sintetizar en los siguientes aspectos:

- Cambios en la denominación de los establecimientos. Aunque la tipología predominante ha sido desde el principio la de la casa rural, han existido otras figuras que se fueron incorporando o desapareciendo en los últimos 25 años (Tabla 3). Asimismo, durante este periodo los requisitos exigibles a las casas rurales se han ido modificando a nivel de aspectos cualitativos, cuantitativos, equipamientos y servicios. Además de la casa rural, el apartamento rural, que en el decreto vigente se concibe como una subtipología de la casa rural (casa-apartamento), fue en su momento una de las figuras que experimentó un mayor crecimiento numérico y cambios en su distribución geográfica. Por último, los hoteles rurales se han posicionado como una tipología de alojamiento de gran importancia por disponer de una mayor capacidad.

- Cambios en la concreción de lo que se entiende por medio rural, desde el punto de vista del número de habitantes de los núcleos de población. Desde la publicación del primer decreto se planteó que los alojamientos rurales solo se autorizarían en aquellos municipios que no superaran un determinado número de habitantes. Como se 
observa en la Tabla 3, los requisitos han oscilado, en este sentido, entre los límites de 5.000 y 20.000 habitantes, dependiendo de las tipologías y el momento. En la actualidad, solo se ven privados de albergar alojamientos rurales los municipios que superen los 20.000 habitantes, aunque a efectos de este artículo se ha tenido en cuenta el límite de los 10.000 habitantes por razones ya explicadas.

- Categorización. A partir del año 1998 fueron implantadas categorías específicas para los alojamientos rurales que afectaron, con carácter de exclusividad, a las casas rurales (Tabla 3). Con posterioridad se produjeron otros cambios, hasta llegar a la situación actual en la que se tipifica una categorización en base a estrellas verdes ( 1 a 5 estrellas).

- Capacidad de los alojamientos rurales. La capacidad de los establecimientos rurales ha experimentado cambios (Tabla 3). La tendencia ha sido la de autorizar un mayor número de habitaciones en las casas rurales y suprimir las limitaciones en otras tipologías.

Tabla 3. Síntesis de los principales cambios experimentados por los alojamientos rurales de Extremadura, en el periodo 1992-2015, desde el punto de vista normativo

\begin{tabular}{|c|c|c|c|c|}
\hline Decreto & $\begin{array}{c}\text { Tipologías de } \\
\text { establecimientos }\end{array}$ & $\begin{array}{c}\text { Límite de habitan- } \\
\text { tes }\end{array}$ & Categorías & $\begin{array}{c}\text { Número de } \\
\text { habitaciones }\end{array}$ \\
\hline \multirow[t]{2}{*}{$132 / 1992$} & Casas Rurales & 10.000 & & 6 \\
\hline & Hospederías & 15.000 & & - \\
\hline \multirow{4}{*}{$125 / 1998$} & Casas Rurales & 5.000 & $1-3$ encinas & $2-6$ \\
\hline & Agroturismo & 5.000 & $1-3$ encinas & $2-6$ \\
\hline & Apartamentos & $\begin{array}{r}\text { Situado en el } \\
\text { medio rural (sic) } \\
\end{array}$ & & \\
\hline & Hoteles & 5.000 & Categoría única & $6-30$ \\
\hline \multirow{3}{*}{$87 / 2007$} & Casas Rurales & 5.000 & 2-3 encinas & $3-8$ \\
\hline & $\begin{array}{l}\text { Apartamentos } \\
\text { Rurales }\end{array}$ & 5.000 & & 2 por apartamento \\
\hline & Hoteles Rurales & 5.000 & Categoría única & $10-30$ \\
\hline \multirow{4}{*}{$204 / 2012$} & Casas Rurales & 10.000 & 1-3 encinas & Máximo 9 \\
\hline & $\begin{array}{l}\text { Apartamentos } \\
\text { Rurales }\end{array}$ & 10.000 & $1-3$ encinas & \\
\hline & $\begin{array}{l}\text { Hoteles } \\
\text { Rurales }\end{array}$ & 10.000 & $1-5$ encinas & \\
\hline & Chozos turísticos & 10.000 & & \\
\hline \multirow[t]{2}{*}{$65 / 2015$} & Casas Rurales & 10.000 & $1-5$ estrellas & Máximo 9 \\
\hline & Hoteles Rurales & 10.000 & $1-5$ estrellas & \\
\hline Ley $6 / 2018$ & & 20.000 & & \\
\hline
\end{tabular}

Fuente: Diario Oficial de Extremadura. Elaboración propia

A nivel nacional, también se produce un desarrollo normativo que comienza en la década de los años 80 del siglo $\mathrm{XX}$, aunque hubo algunos casos de comunidades 
autónomas que no lo hicieron hasta que estuvo bien comenzado el siglo XXI (Hernández, 2010). Como consecuencia de ello, se ha asistido a un crecimiento generalizado de la oferta de alojamientos rurales, surgida de esta normativa específica, que algunos autores consideran que se encuentra ya consolidado a mediados de los noventa del siglo XX (Chicharro y Galve, 2009), mostrando cierto retraso con respecto a lo que ocurrió en otros países europeos por motivos diversos (Río del, 2016). No obstante, el principal impulso del sector es posterior, periodo en el que el crecimiento es tan intenso que llega a generalizarse la idea, en diversos trabajos, de que existe un sobredimensionamiento de la oferta que no se corresponde con la demanda, lo que origina bajos niveles de ocupación por la alta competencia y dificultades para alcanzar un umbral de rentabilidad (Grande, 2006). Sin embargo, a pesar de este intenso crecimiento, el turismo rural español se encuentra en un punto intermedio, a medio camino entre los destinos más maduros como Gran Bretaña, Francia o Alemania y de los incipientes, especialmente en la Europa del Este (Cánoves et al. 2012).

\section{Resultados}

Como ya se ha mencionado antes, en este trabajo se van a utilizar, como ámbito de estudio, los 375 municipios extremeños de menos de 10.000 habitantes, si bien se ha establecido una diferenciación entre los municipios menores de 2.000 habitantes, que alcanzan la cifra de 293, y los municipios con una población entre 2.001 y 9.999, cuyo número se eleva a 82 . En el marco de este trabajo se denominará a los primeros de pequeño tamaño y a los segundos de tamaño intermedio. Esta división se ha llevado a cabo con la finalidad de valorar si se aprecian diferencias entre unos y otros municipios en relación con la distribución de los alojamientos convencionales y rurales. Asimismo, cuando se ofrezcan cifras sobre la capacidad de los alojamientos extrahoteleros se diferenciará entre las aportadas por los campamentos de turismo y por el resto de tipologías de alojamientos extrahoteleros (Apartamentos turísticos y albergues turísticos), con el objetivo de hacer una lectura adecuada, dadas las especiales circunstancias que rodean las características de las plazas que ofertan los campings, la mayor parte de las cuales son en parcelas.

\subsection{Número y capacidad de los alojamientos convencionales hoteleros}

De un primer análisis de las cifras obtenidas en lo que a distribución de alojamientos convencionales a escala regional se refiere, se infiere que en el año 2017 había 146 municipios extremeños de menos de 10.000 habitantes en los que se localizaban estos alojamientos, independientemente de su clase y tipología. Esta cifra se corresponde con el 38,9\% de los municipios que cumplen con dicho requisito demográfico en el año de referencia (Figura 2). La distribución de estos municipios a escala provincial era la siguiente: provincia de Badajoz 67 (42,9\% de los municipios de pequeño tamaño), y provincia de Cáceres $79(36,0 \%)$. En términos comparativos con lo que 
ocurría en el año 2001, se aprecia un sustancial incremento, ya que en este año el número de municipios en los que había alojamientos hoteleros era de tan solo 73 . Dicho crecimiento se traduce en un avance que duplicó la oferta en el periodo objeto de estudio.

Figura 2. Distribución territorial de Alojamientos Hoteleros. Año 2017.

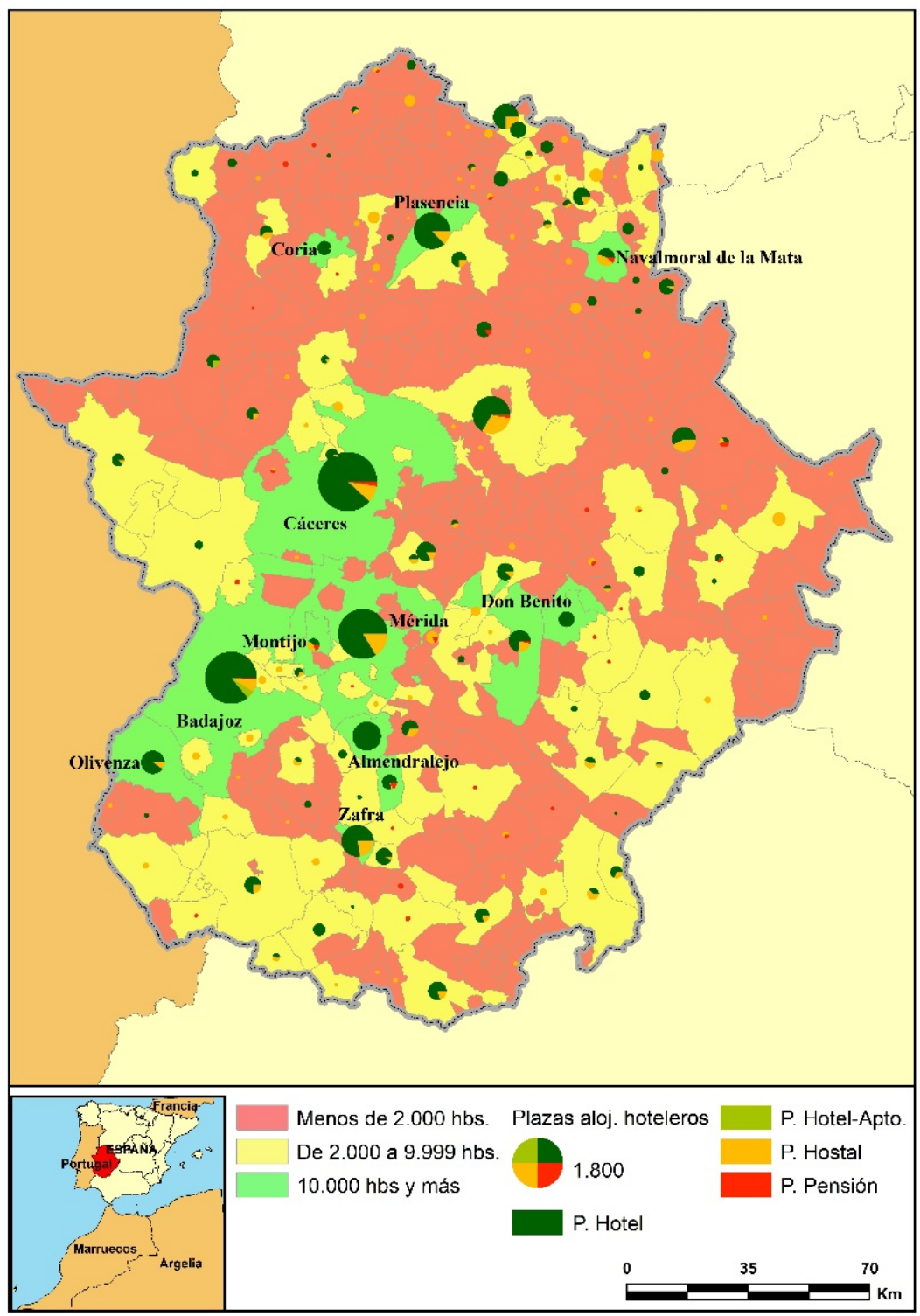

Fuente: Elaboración propia con datos de Dirección General de Turismo (Junta de Extremadura) 
Si se realiza una segunda lectura de los datos, en función de lo que refleja la Tabla 4, se pueden extraer otras valoraciones:

- Municipios de menos de 2.000 habitantes (tamaño pequeño). El número de municipios en los que se ha constatado la presencia de alojamientos hoteleros convencionales se elevó a 78. Esta cifra, tras su traducción porcentual, equivale al $26,6 \%$ de los municipios que cumplen con este requisito poblacional a escala de Extremadura (293), si bien, cuando se analizó la proporción a escala provincial se observaron diferencias entre las dos provincias extremeñas: Cáceres $(29,3 \%)$ y Badajoz (21,2\%). En su conjunto, estos municipios cuentan con 140 establecimientos que ofertan 4.104 plazas. Los alojamientos más representativos son, desde el punto de vista numérico, los hostales de 1 y 2 estrellas que, además, son los que ofertan un mayor número de plazas en su conjunto. Por su parte, los hoteles de 4 estrellas se comportan de forma diferente, ya que a pesar de su escaso número, cuentan con una elevada capacidad debido a que el número medio de plazas por establecimiento es sensiblemente superior. Estas cifras comparadas con la situación del año 2001 evidencian un crecimiento sustancial, tanto en número de establecimientos como de plazas en cualquiera de los alojamientos y categorías seleccionados.

- Municipios entre 2.000 y 9.999 habitantes. En este caso los municipios con presencia de alojamientos hoteleros convencionales se elevarían a 68. Esta cifra se corresponde con el $82,9 \%$ de los municipios que cumplen este requisito poblacional a escala de Extremadura. Se trata, sin duda, de un porcentaje significativamente más elevado que el visto con anterioridad en los municipios de menos de 2.000 habitantes. Una vez más se manifiestan diferencias entre Cáceres y Badajoz, aunque no excesivas (80,7\% en la provincia de Badajoz y $88,0 \%$ en la de Cáceres). Asimismo, la cifra de alojamientos se eleva a 166, cuya capacidad total alcanza las 5.251 plazas. Los alojamientos que aportan un mayor volumen de plazas son los hoteles de 3 estrellas, seguidos de los hostales de 1 y 2 estrellas. De igual modo, es notable la aportación de plazas proveniente de los hoteles de 4 estrellas, a pesar de su escaso número. En relación con la presencia de hoteles de cuatro estrellas en municipios de menos de 10.000 habitantes, hay que señalar el papel que ha adquirido la red de hospederías de Extremadura ( 8 hoteles de cuatro estrellas), cuya puesta en marcha y gestión depende de la Administración regional. Si se contrastan estos datos con los del año 2001 se aprecia un incremento en todas las categorías reflejadas en la Tabla 4, con la excepción de los hoteles de dos estrellas. No obstante, este incremento no es tan significativo como el que se observó en los municipios menores de 2.000 habitantes 
Tabla 4. Alojamientos convencionales hoteleros en Extremadura. Años 2001 y 2017

\begin{tabular}{|c|c|c|c|c|c|c|c|c|c|c|c|c|}
\hline \multirow{3}{*}{ 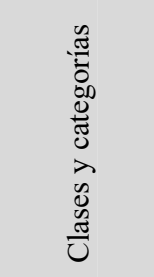 } & \multicolumn{4}{|c|}{$\begin{array}{c}\text { Municipios de menos de } \\
2.000 \text { habitantes } \\
\text { (Pequeño Tamaño) }\end{array}$} & \multicolumn{4}{|c|}{$\begin{array}{c}\text { Municipios de } 2.000 \text { a } 9.999 \\
\text { habitantes } \\
\text { (Tamaño Intermedio) }\end{array}$} & \multicolumn{4}{|c|}{$\begin{array}{l}\text { Municipios de más } 10.000 \text { de } \\
\text { habitantes }\end{array}$} \\
\hline & \multicolumn{2}{|c|}{$\mathrm{N}^{\circ}$} & \multicolumn{2}{|c|}{ Capacidad } & \multicolumn{2}{|c|}{ Número } & \multicolumn{2}{|c|}{ Capacidad } & \multicolumn{2}{|c|}{ Número } & \multicolumn{2}{|c|}{ Capacidad } \\
\hline & 2001 & 2017 & 01 & 2017 & 2001 & 2017 & 2001 & 2017 & 2001 & 2017 & 2001 & 2017 \\
\hline H 5* & 0 & 0 & 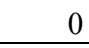 & & 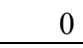 & & 0 & 0 & 1 & $T$ & & $3 / 2$ \\
\hline $\mathrm{H} 4 *$ & 2 & 0 & 176 & 14 & 6 & & 546 & 09 & 14 & 26 & 16 & 3.952 \\
\hline H $3 *$ & 2 & 6 & 21 & 4 & 1 & 1 & 1070 & 1329 & & 24 & 1.725 & 2.378 \\
\hline $\mathrm{H} 2 *$ & 6 & 14 & 242 & 616 & 17 & 1 & 720 & 602 & 3 & 9 & 692 & 906 \\
\hline H $1 *$ & 9 & 6 & & & 7 & & 91 & 1 & & & 8 & 42 \\
\hline HotelA & 0 & 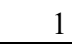 & 0 & & & & 0 & 0 & & & 0 & 112 \\
\hline Hs 2* & 11 & 34 & 284 & 729 & 20 & 3 & 587 & 811 & 12 & 23 & 384 & 662 \\
\hline Hs $1 *$ & 22 & 46 & 505 & & 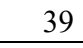 & 56 & 869 & & 4 & 19 & 407 & 526 \\
\hline Pens. & 7 & 18 & 97 & 225 & 10 & 15 & 118 & 166 & 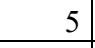 & 11 & 84 & 173 \\
\hline Total & 59 & 140 & 1.753 & 4.104 & 117 & 166 & 4.401 & 5.251 & 90 & 145 & 6.868 & 9.723 \\
\hline
\end{tabular}

Fuente: Elaboración propia con datos de Dirección General de Turismo (Junta de Extremadura)

Por último, aunque la realidad de los municipios de más de 10.000 habitantes no es objeto de análisis en este trabajo, se ha considerado pertinente incluir las cifras que alcanzan en ellos los alojamientos hoteleros para extraer conclusiones adicionales. En este sentido, hay un dato muy importante que indica que si bien se aprecia que los municipios de menos de 10.000 habitantes cuentan con un mayor número de alojamientos convencionales (308 frente a 145 en el año 2017), la capacidad de los alojamientos convencionales ubicados en los municipios de mayor peso demográfico, es superior. Esto se debe a que los municipios de más de 10.000 habitantes acaparan la mayoría de establecimientos de alta categoría (54 establecimientos de 3 a 5 estrellas, frente a 43) que se caracterizan, a su vez, por tener un tamaño superior en lo que a número de plazas se refiere. A modo de ejemplo se puede citar el hecho de que de los 22 hoteles extremeños (17 hoteles de 4 estrellas y 5 hoteles de 3 estrellas) que superan las 150 plazas de capacidad, 18 se ubican en municipios que superan los 10.000 habitantes: Badajoz (6), Almendralejo (1), Mérida (4), Olivenza (1), Cáceres (5) y Don Benito (1). Por su parte, los 4 restantes se ubican en municipios que están por debajo de esa cifra: Puebla de Sancho Pérez, El Gordo, Trujillo y Valdastillas. En su conjunto, estos 22 establecimientos vienen a sumar casi el $24 \%$ de la capacidad que ofertan los alojamientos convencionales.

\subsection{Número y capacidad de los alojamientos convencionales extrahoteleros}

Del examen elaborado, a partir de los datos obtenidos, se aprecia que en 74 municipios extremeños de menos de 10.000 habitantes se localizaban alojamientos 
extrahoteleros durante el año 2017 (Figura 3). Esta cifra solo representa al 19,7\% de los municipios que cumplen con este requisito poblacional, no obstante, en comparación con los datos del año 2001 (solo 17 municipios), hay que hablar de un crecimiento muy significativo que se nutre, en su esencia, del importante crecimiento de apartamentos y albergues.

Tabla 5. Alojamientos convencionales extrahoteleros en Extremadura. Años 2001 y 2017

\begin{tabular}{|c|c|c|c|c|c|c|c|c|}
\hline \multirow{3}{*}{ 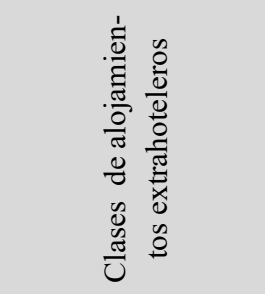 } & \multicolumn{4}{|c|}{$\begin{array}{c}\text { Municipios de menos de } 2.000 \\
\text { habs. }\end{array}$} & \multicolumn{4}{|c|}{ Municipios de 2.000 a 9.999 habs. } \\
\hline & \multicolumn{2}{|c|}{ Número } & \multicolumn{2}{|c|}{ Capacidad } & \multicolumn{2}{|c|}{ Número } & \multicolumn{2}{|c|}{ Capacidad } \\
\hline & 2001 & 2017 & 2001 & 2017 & 2201 & 2017 & 2001 & 2017 \\
\hline $\begin{array}{l}\text { Apartamentos } \\
\text { turísticos }\end{array}$ & 1 & 38 & 10 & 406 & 3 & 34 & 57 & 796 \\
\hline $\begin{array}{l}\text { Campamentos de } \\
\text { turismo }\end{array}$ & 9 & 13 & 3.019 & 3.598 & 9 & 11 & 3.578 & 4.203 \\
\hline Albergues & 2 & 20 & 231 & 765 & 1 & 15 & 55 & 585 \\
\hline Total & 12 & 71 & 3.260 & 4.769 & 13 & 60 & 3.690 & 5.584 \\
\hline
\end{tabular}

Fuente: Elaboración propia con datos de Dirección General de Turismo (Junta de Extremadura)

La distribución de los municipios que poseían alojamientos extrahoteleros en el año 2017, a escala provincial, muestra sustanciales diferencias entre los datos asociados a la provincia de Badajoz, cuyo número era de tan solo $5,(3,2 \%$ de los municipios de menos de 10.000 habitantes de la provincia), y los de Cáceres, con 39 (17,8\% de los municipios de menos de 10.000 habitantes de la provincia). No obstante, del análisis detallado del reparto de las diferentes figuras de alojamientos extrahoteleros en el año 2017, en comparación con el año 2001, se pueden extraer las siguientes lecturas:

- Apartamentos turísticos. Su presencia es muy escasa en los municipios de pequeño tamaño y tamaño intermedio. Por este motivo solo 24 municipios de menos de 2000 habitantes y 17 de entre 2.000 y 9.999 cuentan con algún alojamiento de este tipo, distribuyéndose en mayor proporción por localidades de la provincia de Cáceres. Asimismo, el número de alojamientos (71), y su capacidad, no son muy relevantes (1202), aunque es innegable que se ha producido un incremento en el periodo objeto de estudio. No cabe hablar, por tanto, de especialización en este tipo de establecimiento por parte de ninguno de los grupos de municipios antedichos. Todo lo contrario de lo que se viene observando en dos localidades de más de 10.00 habitantes con un importante patrimonio histórico (Cáceres y Mérida), en las que se ha venido produciendo un crecimiento espectacular en este sentido. 
Figura 3. Mapa de distribución territorial de los Alojamientos Extrahoteleros. Año 2017

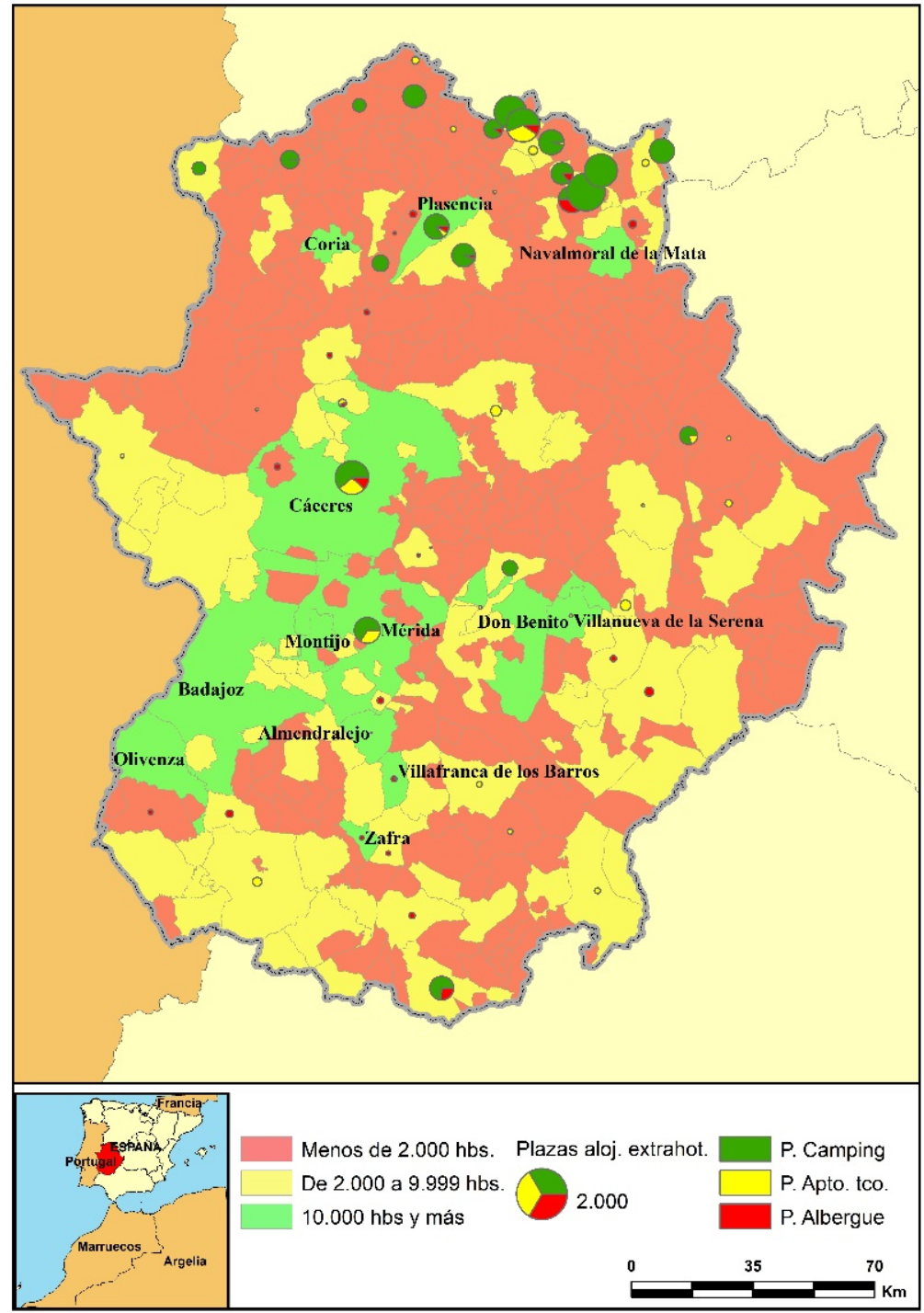

Fuente: Elaboración propia con datos de Dirección General de Turismo (Junta de Extremadura)

- Campamentos de turismo. Por sus especiales características y diseño, la capacidad de los campamentos de turismo no es directamente comparable con la del resto de alojamientos hoteleros, extrahoteleros y rurales. El número actual de campings en municipios de menos de 10.000 habitantes se eleva a 24, ofertando un volumen total de 7.801 plazas. Estas cifras reflejan un avance moderado desde el año 2001 al 2017: +6 nuevos campings y +1.204 plazas. Desde el punto de vista de la 
distribución a escala provincial, la práctica totalidad de estos alojamientos se ubican en municipios de la provincia de Cáceres, pues solo uno está situado en un municipio de la provincia de Badajoz. Asimismo, dentro de la provincia de Cáceres se aprecia una alta concentración en áreas de montaña: comarca de la Vera (8), Valle del Ambroz (4), Valle del Jerte (3) y Sierra de Gata-Hurdes (4).

- Albergues turísticos. El número de albergues es escaso en Extremadura, tanto en municipios pequeños (17) como intermedios (15), distribuyéndose en una mayor proporción por municipios cacereños. No obstante, hay que precisar que en el periodo objeto de estudio se ha producido una expansión espectacular de esta tipología de alojamiento, al pasar de 3 a 35 el número de albergues y de 286 a 1.350 las plazas.

\subsection{Número de alojamientos rurales}

Tras un intenso proceso de crecimiento, la situación actual de los alojamientos rurales en Extremadura evidencia que han conseguido extenderse por un gran número de municipios de la región. En el año 2017, los alojamientos rurales estaban presentes en 229 localidades, lo que se traduce en el hecho de que el 61,0\% de los municipios de menos de 10.000 habitantes cuenten con al menos un alojamiento rural (Figura 4). Este porcentaje experimenta variaciones si se considera la distribución de los alojamientos rurales a escala provincial. En el caso de la provincia de Cáceres el 69,8\% de los municipios de menos de 10.000 habitantes cuenta con algún alojamiento rural, mientras que en Badajoz este porcentaje disminuye sensiblemente hasta el $48,7 \%$. Estas cifras, en relación a la situación del año 2001, reflejan un crecimiento exponencial. En el año 2001 el número de municipios con alojamientos rurales era de tan solo 36, es decir, una cifra que no superaba ni tan siquiera el $10 \%$ de los municipios menores de 10.000 habitantes.

Por otra parte, a los datos expuestos con anterioridad hay que añadir el hecho de que en 110 municipios extremeños la única tipología de alojamiento existente es la rural, ante la ausencia de alojamientos hoteleros y extrahoteleros. En concreto, estaríamos hablando de 76 municipios de la provincia de Cáceres y 34 de la provincia de Badajoz. Por tanto, en estos municipios el papel de los alojamientos rurales, como vehículo de acercamiento al turismo, es más que evidente.

Tabla 6. Alojamientos rurales en Extremadura. Años 2001 y 2017

\begin{tabular}{|l|r|r|r|r|r|r|r|r|}
\hline \multirow{3}{*}{$\begin{array}{l}\text { Clases de alo- } \\
\text { jamiento rural }\end{array}$} & \multicolumn{4}{|c|}{$\begin{array}{c}\text { Municipios de menos de } 2.000 \\
\text { habs. }\end{array}$} & \multicolumn{4}{c|}{$\begin{array}{c}\text { Municipios de } 2.000 \text { a } 9.999 \\
\text { habs. }\end{array}$} \\
\cline { 2 - 9 } & \multicolumn{2}{|c|}{ Número } & \multicolumn{2}{c|}{ Capacidad } & \multicolumn{2}{c|}{ Número } & \multicolumn{2}{c|}{ Capacidad } \\
\cline { 2 - 9 } & 2001 & 2017 & 2001 & 2017 & 2001 & 2017 & 2001 & 2017 \\
\hline Casas Rurales & 34 & 465 & 306 & 5.250 & 10 & 211 & 98 & 2.720 \\
\hline Hoteles Rurales & 3 & 32 & 77 & 882 & 3 & 31 & 101 & 959 \\
\hline Total & $\mathbf{3 7}$ & $\mathbf{4 9 7}$ & $\mathbf{3 8 3}$ & $\mathbf{6 . 1 3 2}$ & $\mathbf{1 3}$ & $\mathbf{2 4 2}$ & $\mathbf{1 9 9}$ & $\mathbf{3 . 6 7 9}$ \\
\hline
\end{tabular}

Fuente: Elaboración propia con datos de Dirección General de Turismo (Junta de Extremadura) 
Figura 4. Mapa de distribución territorial de los Alojamientos Rurales. Año 2017.

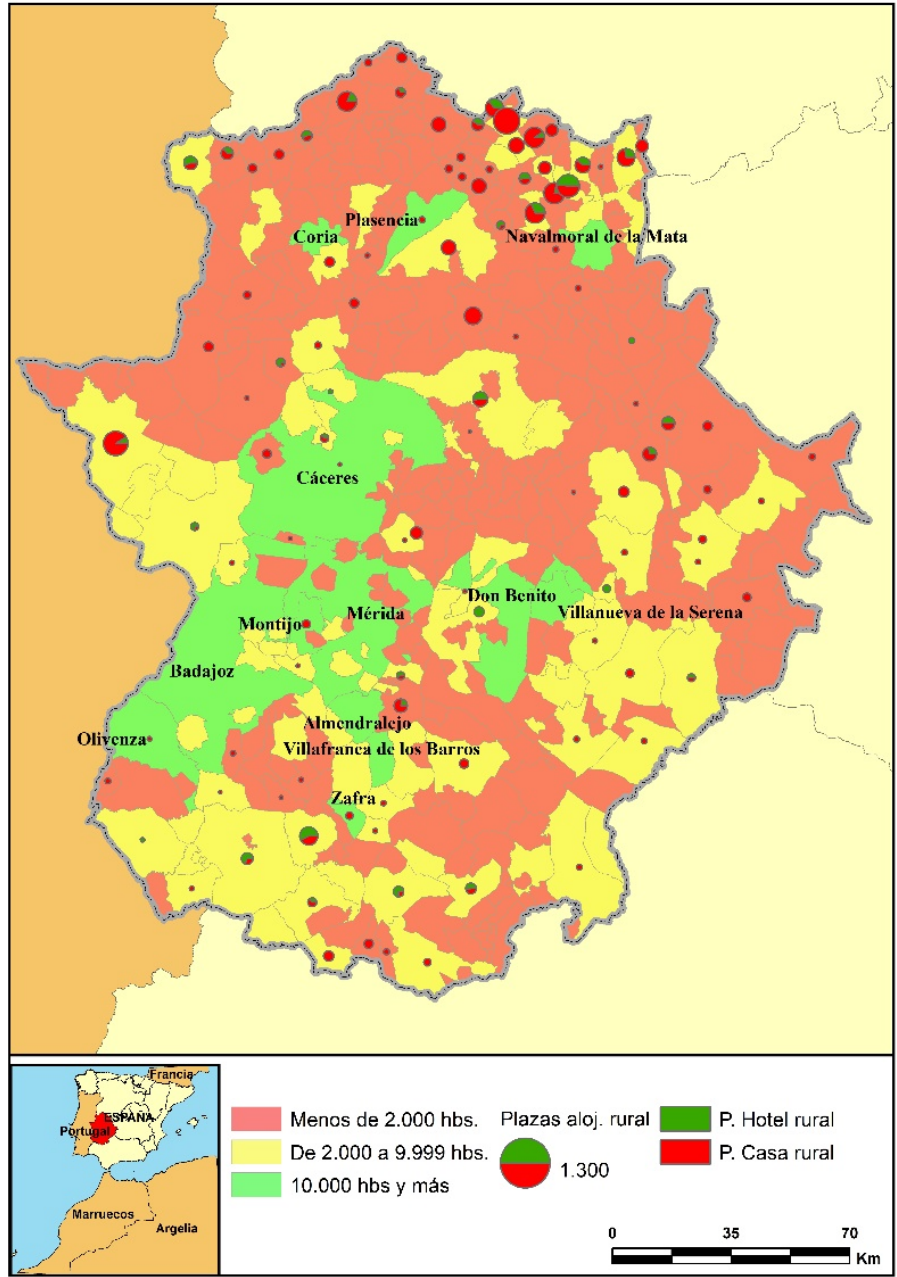

Fuente: Elaboración propia con datos de Dirección General de Turismo (Junta de Extremadura)

No obstante, si estos datos se analizan con más detalle, tras considerar las dos tipologías de alojamientos rurales vigentes, se obtienen las siguientes conclusiones:

- Casas Rurales. Se trata de la tipología de alojamiento rural que ha logrado un mayor desarrollo cuantitativo. A pesar del considerable número alcanzado durante el año 2017 (676 casas), la capacidad total no sobrepasa las 8.000 plazas, resultado de la escasa capacidad media por establecimiento (11,7 plazas), en consonancia con la caracterización de estos alojamientos y lo que expresa la propia regulación. Sin embargo, la principal característica de las casas rurales es su irregular distribución 
geográfica, tanto a escala municipal como supramunicipal (comarcal y provincial). En este sentido, hay que hablar de municipios y comarcas en los que se produce una elevada concentración de esta tipología de alojamientos, frente a otros en los que su número es escaso o casi nulo. A nivel provincial, concretamente, la provincia de Cáceres acumula un número de alojamientos muy superior frente al que presenta la de Badajoz. Por último, un aspecto a destacar es su mayor presencia en los municipios con una población inferior a 2.000 habitantes. En este sentido, hay que señalar que el número de casas rurales localizadas en municipios de menos de 2.000 habitantes, llega a duplicar la cifra de casas rurales existentes en los municipios de tamaño intermedio. Por último, el crecimiento experimentado por las casas rurales en el periodo 2001-2017 ha sido espectacular, al pasar de 44 a 676.

- Hoteles Rurales. Su número es sensiblemente inferior respecto al de casas rurales, si bien esta figura tiene un indudable protagonismo en lo que a capacidad se refiere al sumar más de 1.800 plazas. En este caso, la distribución de alojamientos y, consecuentemente, su capacidad, está repartida de forma equitativa entre municipios de pequeño y medio tamaño. Asimismo, se aprecia un incremento más que notable del número de hoteles rurales en el periodo analizado, cuya deriva ha sido pasar de 6 establecimientos en el año 2001 a 63 en el 2017.

Tabla 7. Distribución del número de alojamientos y plazas por tamaño de los municipios. Año 2017

\begin{tabular}{|l|c|c|c|c|c|c|c|c|}
\hline \multirow{2}{*}{ Extremadura } & \multicolumn{2}{|c|}{$<2.000$ habs. } & \multicolumn{2}{c|}{$\begin{array}{c}\text { De } 2.000 \mathrm{a} \\
9.999 \text { habs. }\end{array}$} & \multicolumn{2}{c|}{$>10.000$ habs. } & \multicolumn{2}{c|}{$\begin{array}{c}\text { Total } \\
\text { Extremadura }\end{array}$} \\
\cline { 2 - 9 } & $\mathrm{N}^{\mathrm{o}}$ & Plazas & \multicolumn{1}{|c|}{$\mathrm{N}^{\mathrm{o}}$} & Plazas & $\mathrm{N}^{\mathrm{o}}$ & Plazas & $\mathrm{N}^{\mathrm{o}}$ & Plazas \\
\hline Total & 709 & 15.005 & 469 & 14.514 & 222 & 11.770 & 1.400 & 41.289 \\
\hline Total sin camping & 696 & 11.407 & 458 & 10.311 & 219 & 10.476 & 1.373 & 32.194 \\
\hline
\end{tabular}

Fuente: Elaboración propia con datos de Dirección General de Turismo (Junta de Extremadura)

Un dato no esperado es el hecho de que los municipios de menos de 2.000 habitantes contaran en el año 2017, no solo con el número más elevado de alojamientos de forma conjunta $(50,6 \%)$, sino de plazas totales $(36,3 \%)$. Los 709 alojamientos distribuidos por estos municipios no solo superan ampliamente el número de establecimientos localizados en los municipios de mayor tamaño (469 alojamientos en municipios de 2.000 a 9.999 habitantes y 222 alojamientos en municipios de más de 10.000 habitantes), sino que también lo hacen en el número de plazas totales. Esta circunstancia se repite, aunque atenuada, si se eliminan las plazas que suman los campamentos de turismo, tal y como se puede observar en la Tabla 7. 


\section{Conclusiones}

Extremadura es una región en la que predominan los municipios de tamaño pequeño e intermedio que, además, se caracterizan por una densidad de habitantes muy baja. Como consecuencia de ello, la aprobación de una normativa específica para los alojamientos rurales, cuya finalidad era la de generar actividad turística en municipios de escaso peso demográfico, fue muy bien acogida en su momento. En este sentido hay que señalar que forma parte de la cultura regulatoria de los alojamientos rurales el que se ubiquen en municipios que no superen un número de habitantes determinado. En Extremadura este techo ha ido variando de los 5.000 a los 10.000 , experimentando un recientísimo cambio hasta los 20.000 habitantes. Como en este artículo se ha tenido en cuenta el límite de los 10.000 habitantes, techo máximo vigente en el año 2017, hay que señalar que el 96,6\% de los municipios de la región eran susceptibles de contar con un alojamiento rural.

Cuando los alojamientos rurales fueron regulados, ya estaban reglamentadas otras figuras de alojamientos que acumulaban una larga trayectoria y cuya caracterización respondía a criterios que a los efectos de este artículo se han denominado convencionales (hoteleros y extrahoteleros). Dichos alojamientos son enormemente diversos en lo que a clases y categorías se refiere y su implantación en el territorio extremeño goza de una cultura más dilatada. Tras el detallado análisis de la localización de la oferta de alojamientos de Extremadura en el contexto actual, lo que ha supuesto manejar bases de datos integradas por numerosas variables provenientes de la Dirección General de Turismo de la Junta de Extremadura, se han obtenido unos resultados que reflejan una compleja realidad territorial que es necesario interpretar en el contexto actual:

- Alojamientos hoteleros. El 38,9\% de los municipios (146 de 375) con una población inferior a los 10.000 habitantes contaban con alojamientos hoteleros convencionales en el año 2017. Este porcentaje varía si se establece una diferenciación entre municipios de pequeño tamaño e intermedio, en cuyo caso se aprecia que el $26,6 \%$ de los municipios menores de 2.000 habitantes, y el $82,9 \%$ de los que poseen entre 2.000 y 9.999, cuentan con alojamientos hoteleros. Estas cifras evidencian una tendencia que favorece a los municipios de tamaño intermedio, aunque en el periodo objeto de estudio (2001-2017) no se puede ignorar que los municipios de pequeño tamaño han sido muy dinámicos.

- Alojamientos extrahoteleros. Por su parte, en 74 municipios extremeños de menos de 10.000 habitantes se localizan alojamientos extrahoteleros, ya sean campamentos, albergues o apartamentos turísticos. Esta cifra se corresponde con casi el 20,0\% de los municipios menores de 10.000 habitantes. En el caso de que se tengan en cuenta por separado los dos tramos establecidos (pequeño e intermedio tamaño) los porcentajes se comportan de la siguiente manera: el 15,0\% de los municipios menores de 2.000 habitantes cuentan con algún alojamiento extrahotelero, mientras que en los municipios de entre 2.000 y 9.999 habitantes el porcentaje se eleva al $37,8 \%$. 
- Alojamientos rurales. Por último, los alojamientos rurales se distribuyen por el $61,0 \%$ de los municipios de menos de 10.000 habitantes (229 municipios). Este porcentaje es del $59,7 \%$ si solo se tienen en consideración los municipios de pequeño tamaño y del $65,8 \%$ si se toma como referencia el número de municipios de tamaño intermedio. Además, se ha constatado una especialización en 110 municipios en lo que la presencia de alojamientos rurales se refiere, ya que en estos no existe ninguna otra tipología de alojamientos.

En síntesis, si se establece una comparativa entre los tres bloques de alojamientos (hoteleros, extrahoteleros y rurales) se corrobora que el peso demográfico influye en la distribución de las diferentes tipologías de alojamientos y, consecuentemente, en la capacidad. En este sentido, se confirma que los municipios de menos de 2.000 habitantes son los que cuentan con un mayor número de establecimientos y plazas de alojamientos rurales. Estos mismos municipios son, a su vez, los que cuentan con una menor presencia de alojamientos hoteleros y, por lógica, de plazas de estas características. Menos concluyentes resultan los datos referidos a los alojamientos extrahoteleros. Por último, lo expuesto antes explica que en un $61 \%$ de los municipios menores de 2.000 habitantes se localicen alojamientos rurales, mientras que los alojamientos hoteleros solo van a estar presentes en un $26,6 \%$ de dichos municipios y los extrahoteleros en un $15 \%$.

La lectura que se puede extraer de estos datos evidencia que los pequeños municipios extremeños juegan un papel muy importante en el desarrollo turístico regional, contribuyendo a que la actividad turística no sea exclusiva de los escasos municipios que cuentan con un mayor número de habitantes, circunstancia a la que habría que añadir los consiguientes beneficios positivos que de ello se derivan en términos de desarrollo. Esta circunstancia debe ser tenida en cuenta por el conjunto de actores públicos de carácter turístico de cara a la planificación y diseño de estrategias y políticas de promoción.

\section{Bibliografía}

Alvarado, E. (2006): El turismo en el desarrollo rural. Norba geografía, 11, 129-163.

Campesino, A-J., Rengifo, J.I. y Sánchez, M. (1997): Análisis de la coyuntura turística extremeña en 1995. En AECIT (Coord.): La actividad turística española en 1995. Madrid, Aecit, 441-452.

Campesino, A-J., Rengifo, J.I. y Sánchez, M. (2016): Extremadura. En Alén y Calero (Dir.): La actividad turística española en 2015. Madrid, Editorial Síntesis, 249-258.

Cánoves, G., Herrera, A, L. y Blanco, A. (2005): Turismo rural en España: un análisis de la evolución en el contexto europeo. Cuadernos de Geografía, 77, 41-58.

Cánoves, G., Garay, Ll. y Duro, J.A. (2012): Turismo rural en España: Avances y retrocesos en los últimos veinte años. Papers de turisme, 51, 7-21.

Carazo, L. (1982): El turismo rural como recurso de la población agraria. Revista de Estudios Agrosociales, 120, 117-130. 
Chicharro, E. y Galve, A. (2009): Alojamientos rurales en España: entre el crecimiento acelerado y el peligro de una sobredimensión. Serie Geográfica, 15, 125-137.

European Commission (1997): Rural Development. Office for Official Publications of the European Communities.

Hernández, R. M. (2010): La normativa española sobre turismo rural. Estudios Turísticos, $183,25-41$

Grande, J. (2006): Evolución del turismo rural en España y las nuevas oportunidades del turismo de naturaleza. Estudios Turísticos, 169-170, 85-102

OCDE (1994): Creating rural indicators for shaping territorial policy. Paris.

Rengifo, J.I. y Sánchez, J. M. (2016): Balance del crecimiento del turismo rural en Extremadura (1992-2015): dos décadas de éxitos y decepciones. En Santos X.M., Taboada de Zuñiga P. y López L. (Edit.): X CITURDES, Congreso internacional sobre turismo rural e desenvolvimiento sustentável. Universidad de Santiago de Compostela, 275-289.

Rengifo, J.I., Sánchez, J. M. y Sánchez, M. (2013): Análisis del desarrollo del turismo rural en la provincia de Cáceres en los inicios del S.XXI. Pasos: Revista de Turismo y Patrimonio Cultural, 11 (4), 615-630.

Río del, J. (2016): El turismo rural en Castilla y León: 1996-2017. Pasado, presente y futuro. Gran Tour, 13, 105-131.

Sancho, J. y Reinoso, D. (2012): La delimitación del ámbito rural: una cuestión clave en los programas de desarrollo rural. Estudios Geográficos, Vol. LXXIII, 273, 599-624.

Sánchez, J.M., Gurría J.L., Leco F. y Pérez M. N. (2001): SIG para el desarrollo turístico en los espacios rurales de Extremadura. Estudios Geográficos, 62 (243), 335-368.

Sánchez, J.M. (2009): El sistema de información geográfica como herramienta de análisis turístico. Una aplicación para la localización idónea de alojamientos rurales en la provincia de Cáceres mediante análisis multicriterio. Estudios Turísticos, 182, 71-94.

Sánchez, J. M., Sánchez, M. y Rengifo, J.I. (2013): La evaluación del potencial para el desarrollo del turismo rural. Aplicación metodológica sobre la provincia de Cáceres. Geofocus. Revista Internacional de Ciencia y Tecnología de la Información Geográfica, 13 (1), 99-130.

Sánchez, M., Sánchez, J.M. y Rengifo, J.I. (2014): Methodological approach for assessing the potential of a rural tourism destination: An application in the province of Cáceres (Spain). Current issues in tourism, 19 (11), 1084-1102.

Sánchez, M y Rengifo, J.I. (2016): Análisis crítico de dos destinos extremeños de turismo rural: ¿turismo rural o turismo en el medio rural? En Santos, X.M., Taboada, P. y López L. (Edit.): X CITURDES, Congreso internacional sobre turismo rural e desenvolvimiento sustentável. Universidad de Santiago de Compostela, 305-320.

Sánchez, J. M., Sánchez, M. y Rengifo, J.I. (2017): Análisis del equilibrio entre el potencial turístico y la oferta de alojamientos en turismo rural mediante técnicas de estadística espacial. Una aplicación a la provincia de Cáceres (España). Cuadernos de Turismo, 39, 547-576. 